\title{
Solid Membrane Electrode Assembly for on Board Detection of Peroxides Based Explosives Jonathan C. Mbah ${ }^{a^{*}}$, Scott Steward ${ }^{\mathrm{b},}$ Nosa O. Egiebor ${ }^{\mathrm{c}}$ \\ ${ }^{a}$ Department of Chemical Engineering, Florida Institute of Technology, Melbourne, FL 32901, USA \\ ${ }^{b}$ Naval Explosive Ordnance Disposal Technology Division- Explosive Detection Equipment Program, Indian Head, MD 20640, USA \\ ${ }^{c}$ Department of Chemical Engineering, University of Mississippi, MS 38677, USA
}

\begin{abstract}
The threat to human populace from the use of improvised explosive devices (IEDs) is increasing at an alarming rate. Many devices for IEDs detection lacks sensitivity, reliability, portability: a major issue to be addressed. In this work, we fabricated a disposable solid phase electrolyte/electrode (SPEE) interfaces for the detection of trace quantities of peroxide based explosives, namely, triacetone triperoxide (TATP) and hexamethylene triperoxide diamine (HMTD) which were synthesized without recrystalization, and their precursors: $\mathrm{H}_{2} \mathrm{O}_{2}$ and acetone via electrochemical technique. The SPEE device was able to detect $8.66 \pm 1.048 \mathrm{ng}$ of TATP at its vapor pressure of $4.80 \mathrm{~Pa}$ at $298 \mathrm{~K}$. However, the device only gives good result during the first time usage. As suggested by the electrochemical cell impedance test, mass transfer limitations caused by the solution and charge transfer resistances at the electrode interface impedes reaction kinetics. Nevertheless, the ease of fabrication, response and speed make SPEE sensor an excellent choice and offer opportunity for the development of miniature sensors for screening of IEDs and its precursors at various screening sites.
\end{abstract}

Keywords: Amperometric detection; HMTD; TATP; Metal bromide; Improvised explosives

* Corresponding Author's Information: Email: Joneschinwe2003@yahoo.com (Jonathan C. Mbah); Address: Chemical Engineering, Florida Institute of Technology, $150 \mathrm{~W}$. University Blvd, Melbourne, FL 32901, U.S.A.; Fax: (321)674 7565; Ph: (321)674 8068 


\section{Introduction}

Technologies for explosives detection are often required to have a number of attributes which include that they be low cost, portable and specific. At the most fundamental level sensors contain an immobilized active species which can selectively recognize a material of interest. A transduction step which can be optical, electrochemical or some other technique is then required to convert the recognition event into a measurable change, such as the production of electrons which can be measured at an electrode. For example, the principle of chemical sensors is to have a chemical which undergoes a selective chemical reaction with an explosive vapor, leading to an observable product such as a change in color or conductivity. However, sensitivity is often an issue, and many sensors cannot simply detect the low levels of material required. This is particularly true when attempting to detect low vapor pressure material; such as explosives. Work in the field of explosives sensor development needs to focus on improved sensitivity, reliability and reproducibility.

One sensor that should be considered for explosives vapor detection is an electrochemical sensor. Electrochemical sensors can be categorized into potentiometric (measurement of potential difference/voltage); amperometric (measurement of current) and conductimetric (measurement of conductivity) based devices according to the transduction approach adopted [1]. These types of sensor are fast, inexpensive, have high sensitivity, and are a viable option for miniaturization [2]. Electrochemical detection techniques can respond to redox substances; the electrical output results from a flow in electrons or ions caused by the chemical reaction that takes place at the surface of the electrode. Because of this, the redox properties of peroxide explosive substances ideally lend themselves to electrochemical detection. The detection of peroxide based explosives is often difficult because the materials do not fluoresce, have minimal UV absorption and a lack 
of nitro groups - all necessary parameters upon which many traditional detection techniques are based [3]. Electrochemical sensors in this context offer an opportunity to detect peroxide based explosives that would otherwise prove problematic.

The development of single use processes to enable disposable electrochemical sensing has become more desirable for portable explosive sensors. The use of a Nafion (TM) coated, preanodized screen-printed carbon electrode that increased the sharpness of peaks and, therefore, the ease of identification of substituent groups upon the nitro-aromatic compound has been reported [4]. Recovery of TNT from spiked lake water was $95.7 \%$, with this complex matrix only affecting the sample slightly.

Electrochemical instrumentation has evolved broadly since the early work of Nicholson and Shain on electroanalytical chemistry. In this present study, we fabricated an amperometric sensor to detect trace amounts of peroxides based explosives, namely, hexamethylene triperoxide diamine (HMTD) and triacetone triperoxide (TATP), and TATP precursors-hydrogen peroxide $\left(\mathrm{H}_{2} \mathrm{O}_{2}\right)$ and acetone. Detection of peroxides, $\mathrm{H}_{2} \mathrm{O}_{2}$, using electrochemistry is not a new concept $[5,6]$. In fact, an effective electrode was recently fabricated to detect 6 ppbv of $\mathrm{H}_{2} \mathrm{O}_{2}$ using a prussian blue based electrode [7]. Also, previous work had attempted to electrochemically detect TATP using a solid-state sensor coated with a layer that selectively forms a bond with the vapor [8]. The exact limit of TATP detection was not established in that study.

Cyclic voltammetry is the most popular and convenient electrochemical technique, and usually the first experiment of this kind to be performed. It owes its versatility to its ability to deduce reaction mechanisms with relatively low cost equipment and quick experimentation, and together with chronoamperometry establishes undisputable technique that find useful in sensing trace amounts of explosive materials. 
Staircase voltammetry becomes useful where surface confined effects are important. In this mode, the data is sampled throughout the duration of the step and averaged thereby eliminating the differences between a staircase and a true ramp. Typically, in staircase voltammetry, a current reading is acquired immediately prior to the next step [9]. This method of sampling discriminates against any capacitive or surface bound reactions. The current due to any capacitive charging or faradaic current confined to the surface, decays in the initial part of the step and does not contribute to the measured current. The limiting currents which are proportional to concentration of analyte under study, resulting from electroactivities, are studied by double step chronoamperometry.

In this present study, staircase cyclic voltammetry (CV) and chronoamperometry techniques were utilized to investigate the ability to detect trace amounts of some peroxide based explosives and their precursors, namely, TATP and HMTD, acetone, and $\mathrm{H}_{2} \mathrm{O}_{2}$.

\section{Materials and Methodology}

\subsection{Instrumentation and Reagents}

Amperometric measurements (chronoamperometry and voltammetry) were performed with a 1000 Potentiostat-Galvanostat-ZRA, electrochemical interface analyzer (Gamry Instruments, USA) along with carbon patterned electrode transducers and a $2 \times 10^{-5} \mathrm{~m}^{3}$ electrochemical cell from Pine Instruments. The patterned transducer cards consists of disc screen printed conductive carbon inks as the indicator and auxiliary electrodes, and screen printed silver/silver chloride, $\mathrm{Ag} / \mathrm{AgCl}$, inks as the reference electrodes. Overall card dimensions are $15 \times 61 \times 0.36 \mathrm{~mm}$. The thickness of the carbon, silver, and insulating layers is typically 7 to $13 \mu \mathrm{m}$. 
Stock solutions of 30 and $50 \mathrm{w} \% \mathrm{H}_{2} \mathrm{O}_{2}$ standard solution were purchased from ACROS ORGANICS and used as received, agarose type I-A low electroendosmosis (EEO) - a movement of liquid through the gel (Sigma-Aldrich), $\mathrm{KBr} \geq 99.99 \%$ (Sigma-Aldrich), strengthening nail polish remover as source of acetone (CVS Pharmacy), TATP and HMTD were prepared inhouse, ultrapure water $\left(0.06 \mu \mathrm{Scm}^{-1}\right.$ or $18.2 \mathrm{M} \Omega$-cm. Neither terminology is exclusive).

\subsection{Preparation of Solid Electrolyte}

Alkali metal bromides, are typical ionic salts, and are fully dissociated at near $\mathrm{pH} 7$ in aqueous solution. For this reason, $\mathrm{KBr}$ serves as source of $\mathrm{Br}^{-}$ions in acidic electrolyte. A $1.0 \times 10^{-4} \mathrm{~kg}$ of agarose I-A was dissolved under stirring in a $5 \times 10^{-6} \mathrm{~m}^{3} 0.2 \mathrm{M} \mathrm{KBr}$ solution. The mixture was slowly heated until all the agarose were solubilized. Immediately, at that state, $1.03 \times 10^{-7} \mathrm{~m}^{3}$ agarose droplets were coated on the electrodes surfaces covering the indicator, auxiliary and reference electrodes. Thus, the solid phase electrode/electrolyte (SPEE) interfaced was fabricated in this manner. The droplet was allowed to cool to room temperature and rapidly solidified to form a thin layer gel covering the three electrodes. It is important when heating the solution to dissolve the agarose I-A that evaporation be avoided so that the molarity of the initial $\mathrm{KBr}$ solution is preserved.

\subsection{Device Fabrication for Alkali Metal Bromide Amperometric Cell}

Voltammetric and chronoamperometric measurements were performed using the Gamry electrochemical interface analyzer mentioned previously. To generate vapors of representative test materials: TATP, HMTD, acetone, bottled $\mathrm{H}_{2} \mathrm{O}$ and $\mathrm{H}_{2} \mathrm{O}_{2}$, each sample was sealed in a $2 \times 10^{-}$ ${ }^{5} \mathrm{~m}^{3}$ air tight vial bottle and allowed to equilibrate for $1.8 \times 10^{3} \mathrm{~s}$. In order to compare the response 
of the peroxides based explosives, equal mass of TATP and HMTD were initially charged into the vial bottle. In our previous finding [10] an $8.66 \pm 1.048 \mathrm{ng}$ TATP has a vapor pressure of 4.80 Pa at $298 \mathrm{~K}$ for TATP synthesized using $\mathrm{HCl}$ catalyst as given by a GC-MS headspace approach. Since our present measurement is based on headspace approach, as in our previous work, this mass of TATP was part of the measurement carried out in this study. Subsequently, the SPEE was introduced into the $2 \times 10^{-5} \mathrm{~m}^{3}$ vial cell through an opening in its cap and sealed in place with tape. Agarose gel matrix facilitates the transport of charges and enables diffusion of materials through it [11-15]. Also, current and potential measurements were performed following $60 \mathrm{~s}$ exposure of the electrode to the vapor. This exposure time was to enable the SPEE to equilibrate with the test vapor. Digital staircase voltammetry and double step chronoamperometry were performed.

\section{Results and Discussion}

\subsection{Amperometry}

The aim of this study is to develop a miniature sensor for peroxide based explosives for application at screening locations. In order to achieve this goal electroactive behavior of some peroxide explosives and their precursor species have been studied. The redox of TATP, HMTD, acetone (contained in the strengthening nail polish remover) and $\mathrm{H}_{2} \mathrm{O}_{2}$ in a $\mathrm{KBr}$ solid electrolyte environment were chosen for this purpose.

Preliminary investigation was conducted in order to set the redox potential by voltammetric experiment as shown in Fig. 1. Subsequently, potential for the double step chronoamperometry was stepped from $+0.70 \mathrm{~V}$ to $+0.96 \mathrm{~V} / \mathrm{Ag} / \mathrm{AgCl}$ where electrode reaction occurs corresponding to the diffusion-limited reduction-oxidation. There was no electroactive specie involved using 
the electrode by itself and as such, no redox peak was observed. When the solid electrolyte, 0.2 $\mathrm{M} \mathrm{KBr}$, was incorporated into the electrolytic cell, the resulting voltammograms produced reduction peaks. In the voltammetry experiment, the fast sweep rate produced more current as expected. This was due to larger concentration gradients formed because of reduced time for the bulk solution to diffuse into the layer of product that forms locally at the surface of the working electrode.

Note the sign convention for $\mathrm{E}$ in Fig. 1 which increases from $-\mathrm{E}$ to $+\mathrm{E}$ to the right of the plot (North American convention is the reverse). In this manner, the forward (oxidation) reaction is favored at potentials positive of the $\mathrm{E}^{0}$ for the couple and gives a positive current. The reverse (reduction) reaction is favored at potentials negative of the $\mathrm{E}^{0}$ for the couple and gives a negative current.

Figure 2 is a current vs. time double step chronoamperometric recording of TATP precursors$\mathrm{H}_{2} \mathrm{O}_{2}$ at two different concentrations and acetone derivative from strengthening nail remover, and water serving as a blank test. The SPEE sensor was able to detect vapors of both precursors but was unresponsive to water vapor at 0.70 and $0.96 \mathrm{~V}$ potential steps. The cathodic currents recorded for these potential steps were indicative of reduction reaction. This suggests that aqueous bromide, $\mathrm{Br}_{2}$ was present initially at potential where no electrode reaction occurred, and was reduced to $\mathrm{Br}^{-}$with the electrode potential steps applied. Bromide ions continued to be generated with time.

However, the rate of chemical reaction is minimized at the limiting current due to decrease in concentration gradients from depletion of reactants at the triple phase boundary of the electrode, analyte and electrolyte, and subsequent equilibration which followed. It must be borne in mind that selective detection of these precursors is crucial at screening points. Accuracy in detection of 
$>10 \mathrm{wt} \% \mathrm{H}_{2} \mathrm{O}_{2}$ precursor used for preparing peroxide explosive devices is desired in an airport screening setting. The ability to selectively and non-invasively detect the presence of $\mathrm{H}_{2} \mathrm{O}_{2}$ vapor in common bottled containers is critical for such screening.

From the result obtained, the SPEE sensor fabricated in this manner has proven its ability to detect with speed and sensitivity, trace vapor of $\mathrm{H}_{2} \mathrm{O}_{2}$ and acetone: the two primary precursors used in the TATP synthesis. From the initial 0.013 moles $\mathrm{H}_{2} \mathrm{O}_{2}$ present, an LOD of $8.8 \mu$ mole was established using the SPEE sensor. It was not feasible to determine the LOD of acetone from mixtures of other 12 ingredients in the strengthening nail polish remover. It must be noted that polish nail remover due to its availability has been used as a source of acetone in the clandestine laboratory to make primary explosives such as TATP and HMTD. Our aim is to test our sensor using this product. It is possible that the signal observed may also be a contribution from other ingredients present. However, any signal observed will serve as a means to indicate the presence of acetone which serves as initial measure to combat act of terrorism. False negatives or positives were absent in repetitions of these experiments using the disposable SPEE sensor.

Figures $3 \mathrm{a}$ and $\mathrm{b}$ are the double step chronoamperometry recorded for vapors of TATP that were synthesized using two different acid catalysts- $\mathrm{HCl}$ and $\mathrm{HNO}_{3}$. The sensitivity of the SPEE sensor in detecting TATP vapor contained in the headspace is evident from the two synthesis types. The analytes concentration in real situations depend on the mass and vapor pressures of the analytes present. The SPEE device was able to detect $8.66 \pm 1.048 \mathrm{ng}$ of TATP at its vapor pressure of 4.80 Pa at $298 \mathrm{~K}$. However, the response is more toward TATP synthesized using $\mathrm{HCl}$ catalyst compared to $\mathrm{HNO}_{3}$ catalyst. This is not surprising considering that the former contains less impurity than the later as reported in literature [16]. As a result, there are more molecules of 
TATP in the vapor phase, and therefore more analyte in the bulk electrolyte: faradaic current is directly proportional to the concentration gradients of the analyte.

A remarkable difference in current direction is evident in the double step chronoamperometry of Figs. 2 and 3. This difference occurred at the first step potential $0.70 \mathrm{~V}$. Anodic currents or oxidations were recorded while detecting TATP vapor in Fig. 3. This is indicated by the negative current resulting from the oxidation of $\mathrm{Br}^{-}$to aqueous bromide, $\mathrm{Br}_{2}$, which ultimately was reversed in the second potential step as given by Eqns. 1 and 2. The fate of $\mathrm{Br}_{2}$ generated in the first potential step was determined by the second potential step, where again $\mathrm{Br}_{2}$ is reduced to $\mathrm{Br}$ -

$$
\begin{aligned}
& 2 \mathrm{Br}^{-} \rightarrow \mathrm{Br}_{2}+2 e^{-} \\
& \mathrm{Br}_{2}+2 e^{-} \rightarrow 2 \mathrm{Br}^{-}
\end{aligned}
$$

This noteable different was present in all the analysis and serves to distinguish between the two plots. The LOD established for TATP synthesized from $\mathrm{HCl}$ catalyst is $8.66 \pm 1.048 \mathrm{ng}$. As mentioned previously, this amount of TATP has a vapor pressure of $4.80 \mathrm{~Pa}$ as given by a GCMS headspace analysis [10]. Qualitative analysis from the reduction processes will determine the issuing products involved. We did not carry out this step. We did not carry out any vapor pressure measurements of TATP synthsized with $\mathrm{HNO}_{3}$.

Figure 4 is the the double step chronoamperometric plot for HMTD at three different amounts using the SPEE sensor. Similar result given by TATP was obtained for HMTD detection. Both the anodic and cathodic faradaic currents were recorded at the first and second potential steps respectively. However, based on the initial equal amount of TATP and HMTD charged into the air tight vial, the response is higher for HMTD compared to TATP as evidenced in Figs. 3 and 4 at all concentration tested. Infact, HMTD exhibited almost an order of magnitude in the current 
density higher than TATP. In light of this pattern seen from the peroxide based explosives when compared to the TATP precursors tested, one can assert with confidence that the SPEE fabricated in this study may become instrumental in the detection of trace amount of TATP, HMTD and peroxide vapors concealed in luggage and handbags at screening ports. The SPEE device has also shown its ability to discriminate between these peroxide explosives from blank water and other ingredients in strenghtening nail polish remover which contained acetone, a necessary precursor in TATP synthesis, and also in detecting varying concentration of $\mathrm{H}_{2} \mathrm{O}_{2}$, another precursor in TATP synthesis. The LOD for HMTD was not determined since the vapor pressure of HMTD is difficult to measure due to its instability. The reproducibility of TATP and its precursors have been verified.

\subsection{Performance of the Solid Phase Electrode/Electrolyte Materials}

Electrochemical impedance spectroscopy (EIS) was used to access the SPEE performances over a wide range of frequencies, 0.2 to $100 \mathrm{kHz}$ with an $\mathrm{AC}$ voltage $(\mathrm{mV} \mathrm{rms})$ and 10 points/decade. Our measurement is conducted in a two-electrode mode, so it has two electrode/electrolyte interfaces. We can assume that each interface has a double layer capacitance and a charge transfer resistance. Also there is cell's equivalent series resistance, $R_{\text {esr }}$, in the solution path between the electrodes. Inserting these facts and assumptions into a model, we get the diagram (inset) shown in Fig. 5. The acronyms, R.E and W.E. in the diagram are respectively given to reference and working (indicator) electrodes, Rc1 and Rc2 are the polarization resistances or charge-transfer resistances at the electrode/solution interface, and constant phase angle CPE Y1 and CPE Y2 elements are used in place of double layer capacitance at the electrode interface that are in parallel with the charge transfer resistances. 
At low frequency, the impedance of the capacitors in the model is very high. Both CPE Y1 and CPE Y2 are in parallel with a resistor shown as inset in Fig. 5 (i). The admittance, Y, value for CPE was $8.39 \times 10^{-7} \mathrm{~S}^{\mathrm{a}} \mathrm{s}$. When the capacitors impedance is high, the resistor's impedance dominates. The corresponding Nyquist plot is shown in Fig. 5 (a). An incomplete semi-circle in Nyquist plots, corresponding to a capacitive behavior, was exhibited in this frequency region. Some literature had identified the cause of such incomplete capacitive semi-circle at high frequency region to be associated with the thin electrolyte layer thickness [17-20]. In this study, the electrode thickness was $5.30 \times 10^{-4} \mathrm{~m}$.

At the lowest frequency in Fig. 5, the impedance magnitude is about $6.0 \times 10^{5} \Omega$. This is the sum of $R_{c} 1, R_{c} 2$, and $R_{e s r}$. The high frequency data never becomes resistive as indicated by the constant value of impedance. From the model, the impedance of $R_{\text {esr }}$ is $268 \Omega$. Since the limiting impedance at high frequency is $R_{\text {esr }}$, we estimated the sum of $R_{c} 1$ and $R_{c} 2$ to be $5.9 \times 10^{5} \Omega$.

With the increase of the faradaic reaction speed, the charge transfer resistance decreases. An attempt to reuse the SPEE after the first time usage failed to yield result similar to the first time usage. As a result EIS study was conducted in order to describe the behavior of the electrochemical system. If the cell lacks faradaic reactions, current leads to capacitive charging of the double layers. Thus, we should consider the double layer capacitance $(\mathrm{Cd})$ when the equivalent circuit is determined as shown in Fig. 5 (b). The low frequency region is dominated by sum $R_{c} 1$ and $R_{c} 2$ with a value $50 \mathrm{k} \Omega$. Also, the high frequency region appeared to be capacitive. The impedance at $50 \mathrm{kHz}$ is about $3.9 \mathrm{k} \Omega$. A capacitance value of about $81.6 \mathrm{nF}$ was obtained. Both CPE Y1 and CPE Y2 as shown in Fig. 5(ii) inset are in parallel with a resistor. The combined admittance, Y, value of CPE was $14.49 \times 10^{-6} \mathrm{~S}^{\mathrm{a}} \mathrm{s}^{\mathrm{a}}$. This value is close to an order of magnitude higher than the value obtained at the first time usage $\left(8.39 \times 10^{-7}{\mathrm{~S} . \mathrm{s}^{\mathrm{a}}}^{\mathrm{a}}\right)$. 
In the final analysis, the entire circuit element for first SPEE usage is quite different from that of the failed SPEE impedance measurement. More also, the impedance and capacitance values of the failed SPEE measurements are not reproducible, and will not produce similar result as the first SPEE usage. So it is recommended to replace the SPEE after each usage just as the case in the disposable paper-based sensor used in colorimetric sensing [21].

The value of $R_{\text {esr }}$ for the failed SPEE experiment is about $5 \mathrm{k} \Omega$ while that for the first SPEE usage experiment is $268 \Omega$. This is the solution resistance which actually determines the transport of reactants and ions to and from the electrode interface. The large $\mathrm{R}_{\mathrm{esr}}$ given by the failed SPEE experiment is an indicative of mass transfer limitation problem. The charge transfer or polarization resistances are much higher in the first SPEE usage experiment compared to the failed experiment. Even though charge transfer resistance decreased with faradaic reaction speed, the values obtained is in order of magnitude higher than that of the failed SPEE. However, it is possible that for the failed SPEE, there were not enough reactant transported to the electrode surface as a result of Warburg impedance resulting from mass transfer limitations (electrode could be covered with reaction products, or adsorbed solution components). In that case, fewer reactants that reached the electrode interface were consumed speedily leading to the low value observed.

The high frequency region is capacitive for the failed SPEE experiment due to the Warburg mass transfer limitations from diffusion processes adjacent to the electrode. In addition, diffusion layer effects cause the low frequency end of the impedance in the Nyquist plot to bend over the real axis, giving rise to a double incomplete semicircle shown in Fig. 5 (b). The shape of the Nyquist plot depicts a slow faradaic reaction which eventually leads to the given shape as faradaic resistance increases with increase in overpotential. More also, change in chemical composition of 
the electrode as a result of previous chemical reaction with new products that may not be electroactive or exhibiting different electrode kinetics than the first SPEE could cause the same effect.

\section{Conclusion}

An effective solid phase electrolyte/electrode (SPEE) interface capable of detecting trace quantities of TATP and HMTD and their precursor was fabricated. The new material has shown its proficiency under ambient conditions to detect trace amount of these materials via amperometric measurements of issuing electrode reactions. While reduction of precursors was experienced for the potential steps in chronoamperometric recording, the two peroxides based explosives tested, TATP and HMTD, both experienced oxidation and reduction. This remarkable difference as noted in their spectra served to distinguish the precursors from TATP and HMTD. Furthermore, the sensing of the precursors in mixtures of other materials as demonstrated by acetone in strengthening nail polish remover, or at different concentrations as shown by $\mathrm{H}_{2} \mathrm{O}_{2}$ was carried out successfully. However, the usability of SPEE is restricted to first time use only due to the failure encountered while attempting to reuse it, and should be replaced at low cost. Further analysis conducted to investigate the electrochemical cell impedance behavior indicated that mass transfer limitations, slow electrode kinetics and covering of the electrode surface by reaction products might have contributed to the failure observed after the first time usage. Given that many sensors cannot simply detect the low levels of explosive vapors required, the SPEE sensor offer an opportunity that can be exploited for development of miniature sensor devices for screening of IEDs and their precursors. 


\section{Acknowledgements}

The authors wish to thank the Technology Management Training Groups Incorporation and the US Office of Naval Research (ONR) for funding award ONR BAA 11-007.

\section{References}

[1] S. Singh, Sensors-an effective approach for the detection of explosives, Journal of Hazardous Materials, 144 (2007) 15-28.

[2] P. Rabenecker, K. Pinkwart, A look behind electrochemical detection of explosives, Propellants Explos Pyrotech. 34 (2009) 274-279.

[3] J. Wang, Electrochemical sensing of explosives, Electroanalysis, 4 (2007) 19:415-423.

[4] J. Wang, Analytical electrochemistry, 3rd ed., Wiley; John Wiley distributor, Hoboken, N.J.; Chichester, (2006).

[5] Munoz R, Lu D, Cagan A et al (2007) Analyst 132:560-565

[6] Karyakin AA (2001), Electroanalysis 13:813-819

[7] John Benedet \& Donglai Lu \& Karel Cizek, Amperometric sensing of hydrogen peroxide vapor for security screening, Anal Bioanal Chem (2009) 395:371-376

[8] F. Dubnikova, R. Kosloff, Y. Zeiri, Z. Karpas, Novel Approach to the Detection of Triacetone Triperoxide (TATP): Its Structure and Its Complexes with Ions, J. Phys. Chem. A 2002, 106, 4951-4956

[9] P.T. Kissinger and W.R. Heineman (Editors), Laboratory Techniques in Electroanalytical Chemistry, Marcel Dekker Inc., New York, Basel; Hong Kong, (1996).

[10] J. Mbah, D. Knott, S. Steward, D. Cornett, Vapor pressure and sublimation enthalpy of triacetone triperoxide by a gas chromatography static headspace approach, International Journal of Energetic Materials and Chemical Propulsion, 14 (4): 321-329 (2015)

[11] W. R. Heineman, P. T. Kissinger, Large-Amplitude Controled-Potential Techniques. In Laboratory Techniques in Electroanalytical Chemistry; P. T. Kissinger, W. R. Heineman, Eds.; Monographs in Electroanalytical Chemistry and Electrochemistry; Marcel Dekker: New York, NY, (1984) pp 51-127.

[12] E. A.Veal, A. M. DAY, B. A. Morgan, Hydrogen Peroxide Sensing and Signaling, Molecular Cell, (2007) 26, 1: 1-14. 
[13] W. Hua, Z. M. Chen, C. Y. Jie, Y. Kondo, A. Hofzumahaus, N. Takegawa, C. C. Chang, K. D. Lu1, Y. Miyazaki, K. Kita, H. L.Wang, Y. H. Zhang, M. Hu, Atmospheric hydrogen peroxide and organic hydroperoxides during PRIDE-PRD'06, China: their concentration, formation mechanism and contribution to secondary aerosols, Atmos, Chem. Phys., (2008) 8, 6755-6773.

[14] H. S. Han, J. M. You, H. Jeong, S. Jeon, Electrochemical sensing of $\mathrm{H}_{2} \mathrm{O}_{2}$ by the modified electrode with pd nanoparticles on multi-walled carbon nanotubes-g-poly(lactic acid), $J$. Nanosci Nanotechnol, (2014)14(6):4050-7.

[15] A. A. Karyakin, Prussian Blue and Its Analogues: Electrochemistry and Analytical Applications, Electroanalysis, (2001) 13:813-819.

[16] M. Fitzgerald, D. Bilusich, Sulfuric, hydrochloric, and nitric acid-catalyzed triacetone triperoxide (TATP) reaction mixtures: an aging study, J Forensic Sci. 2011, Sep;56(5):1143-9. doi: 10.1111/j.1556-4029.2011.01806.x. Epub 2011 May 19.

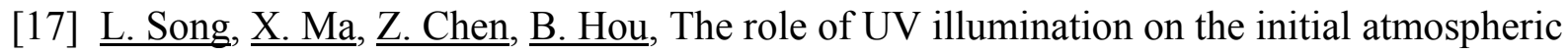
corrosion of 09CuPCrNi weathering steel in the presence of $\mathrm{NaCl}$ particles, Corrosion Science, DOI: 10.1016/j.corsci.2014.07.013.

[18] Cheng Q, Chen Z, The Cause Analysis of the Incomplete Semi-Circle Observed in High Frequency Region of EIS Obtained from TEL-Covered Pure Copper, Int. J. Electrochem. Sci., 8 (2013) $8282-8290$.

[19] Q. Cheng, Z. Chen, The Cause Analysis of the Incomplete Semi-Circle Observed in High Frequency Region of EIS Obtained from TEL-Covered Pure Copper, Int. J. Electrochem. Sci., 8 (2013) $8282-8290$.

[20] A. NASEER, A. Y. KHAN, A study of growth and breakdown of passive film on copper surface by electrochemical impedance spectroscopy, Turk J Chem, 2007.

[21] Miao Xu, Benjamin R. Bunes, and Ling Zang, Paper-Based Vapor Detection of Hydrogen Peroxide: Colorimetric Sensing with Tunable Interface, ACS Appl. Mater. Interfaces 2011, 3, 642-647. 
Author Vitae

\section{Jonathan Mbah, Ph.D.}

Chemical Engineering, Florida Institute of Technology, 150 W. University Blvd,Melbourne, FL 32901, U.S.A.; Fax: (321)6747565; Ph: (321)674 8068

I received my $\mathrm{PhD}$ in Chemical Engineering from the University of South Florida in 2008. I am developing processes in the areas of electrochemical and photoelectrochemical synthesis as applied to energetic materials, carbon dioxide capture and conversion, hybrid batteries/supercapacitors, hydrogen production, and fuel cells.

\section{Scott Steward, Ph.D.}

2008 Stump Neck Road

Indian Head, MD 20640

scott.steward@navy.mil

(301) 744-5142

\section{Employment and Experience:}

April 2009 - Present: Naval Explosive Ordnance Disposal Technology Division (NAVEODTECHDIV), Explosive Detection Technologist.

My duties as the Explosive Detection Technologist are to work ensure that currently available explosive detection equipment (EDE) are objectively evaluated. Additionally, I work to develop new EDE technologies and knowledge, and transition new technologies from development through to fielding.

\section{NosaEgiebor, Ph.D., P.E.}

Senior International Officer, Executive Director of OGE, \& Professor of Chemical Engineering University of Mississippi

Dr. Egiebor holds a PhD degree in Chemical/Metallurgical Engineering from Queen's University in Ontario, Canada. He has over 30 years of direct involvement and leadership in international higher education, both as an administrator and as a faculty member. 


\section{FIGURE CAPTIONS}

Figure 1 (Color online) Cyclic voltammograms $0.2 \mathrm{M} \mathrm{KBr}, \mathrm{E}(\mathrm{V} / \mathrm{Ag}$ vs. AgCl) at 20,25 and $50 \mathrm{mV} / \mathrm{s}$ sweep rates.

Figure 2 ( Color online) Double step chronoamperometric response of water vapor (at $25^{\circ} \mathrm{C}$ ), strengthening nail polish remover vapor, and of vapors from two different concentration of $\mathrm{H}_{2} \mathrm{O}_{2}$ (30 and 50 w\%). Potential step 0.70 and $0.96 \mathrm{~V}(\mathrm{E}$ vs. $\mathrm{Ag} / \mathrm{AgCl})$ with current sampling after $60 \mathrm{~s}$.

Figure 3 (Color online) Double step chronoamperometric response of vapor in the headspace region from varying amounts of TATP synthesized using (a) $\mathrm{HCl}$ and (b) $\mathrm{HNO}_{3}$ catalysts. Potential step 0.70 and $0.96 \mathrm{~V}$ (E vs. $\mathrm{Ag} / \mathrm{AgCl}$ ) with current sampling after $60 \mathrm{~s}$.

Figure 4 (Color online) Double step chronoamperometric response of vapor contained in the headspace region from varying amounts of HMTD. Potential step 0.70 and $0.96 \mathrm{~V}(\mathrm{E}$ vs. $\mathrm{Ag} / \mathrm{AgCl})$ with current sampling after $60 \mathrm{~s}$.

Figure 5 (Color online) Nyquist plot obtained for the first ( a) and second (b) time solid phase electrode/electrolyte (SPEE) interfaced usage with experimental data fitted to CPE with Diffusion model. Inset (i) and (ii) are the equivalent $\mathrm{RC}$ circuit of the two electrode/electrolyte cell for the first and second time SPEE usage 


\section{Figures}

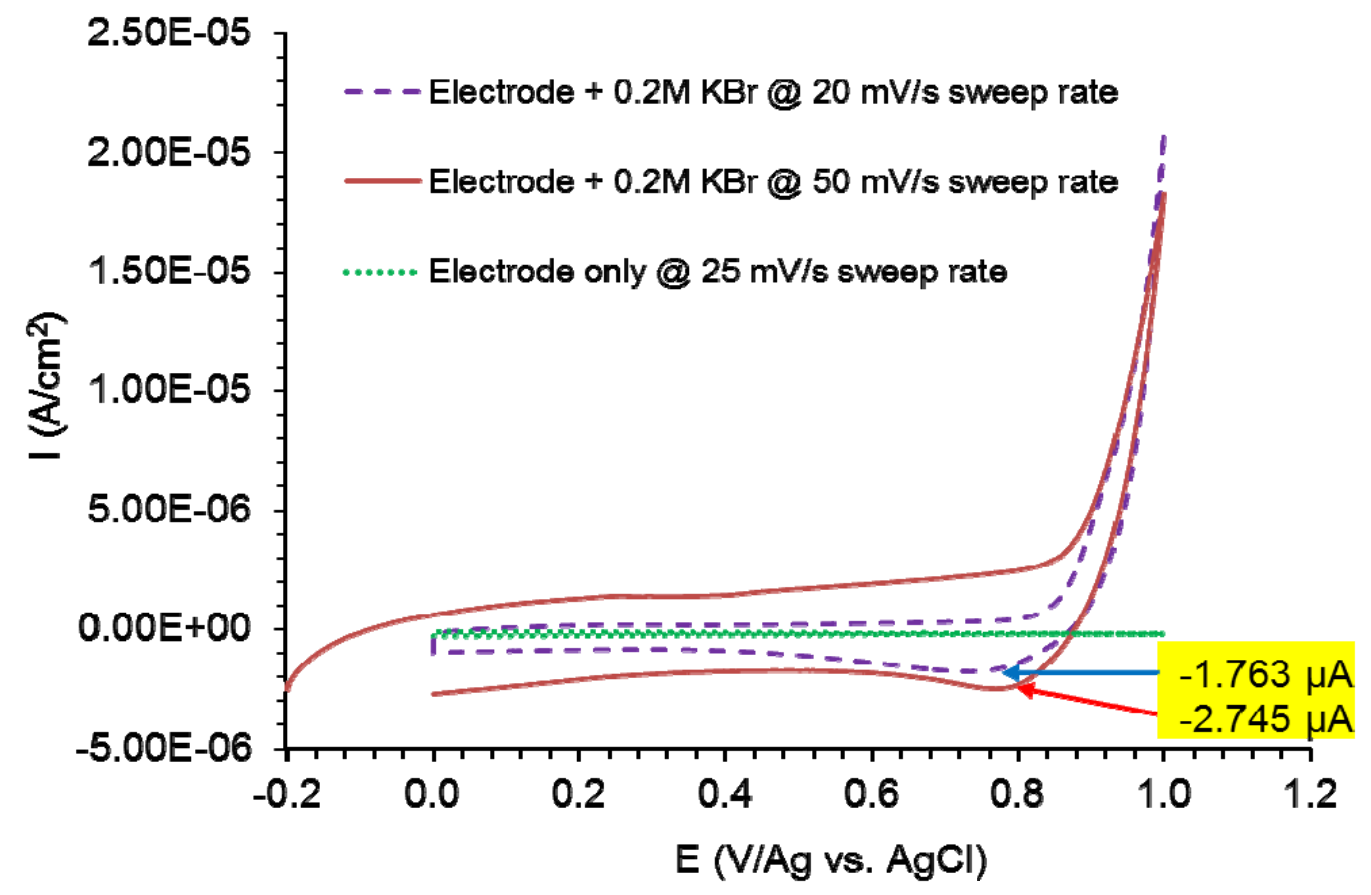

Figure 1. 


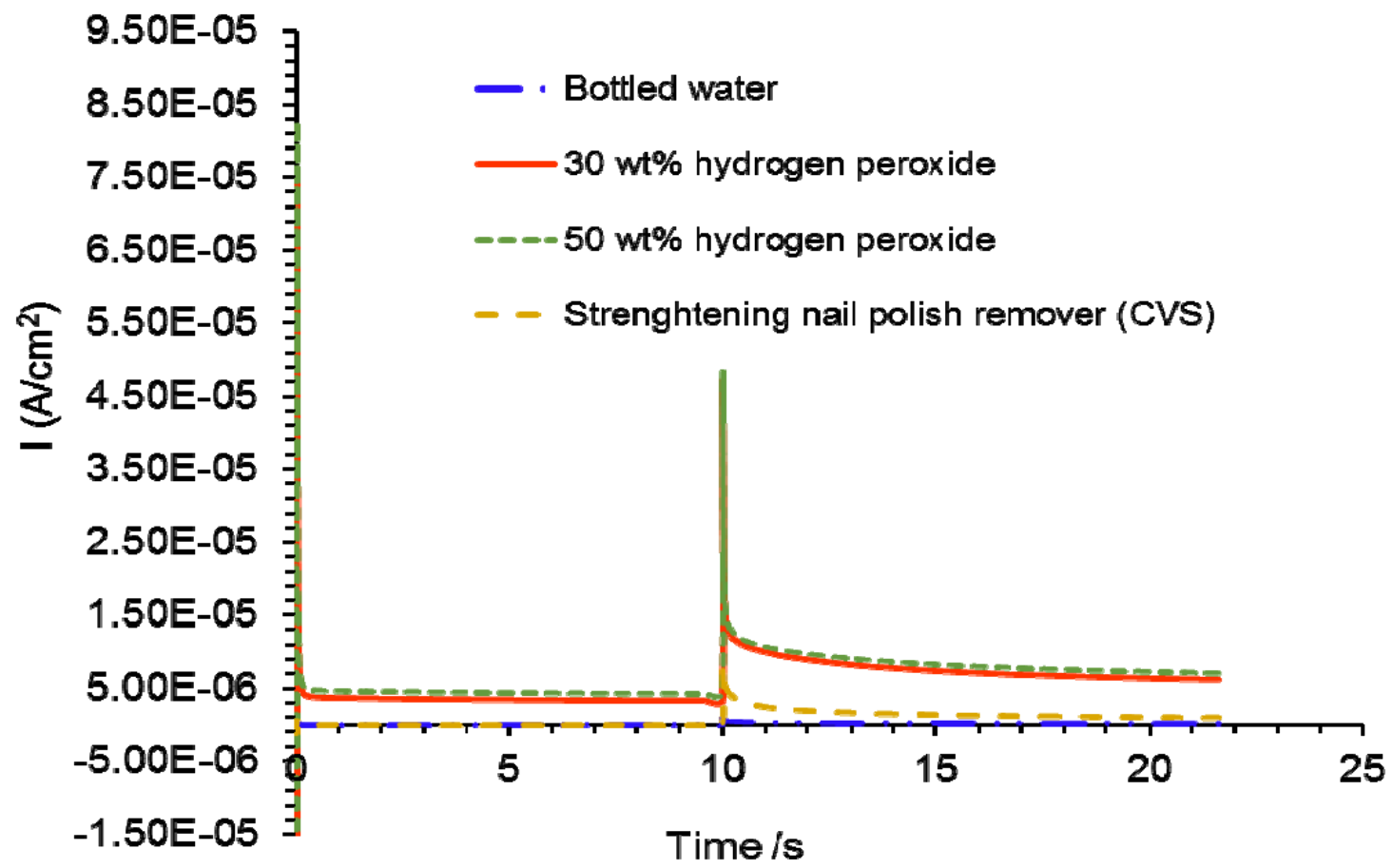

Figure 2. 

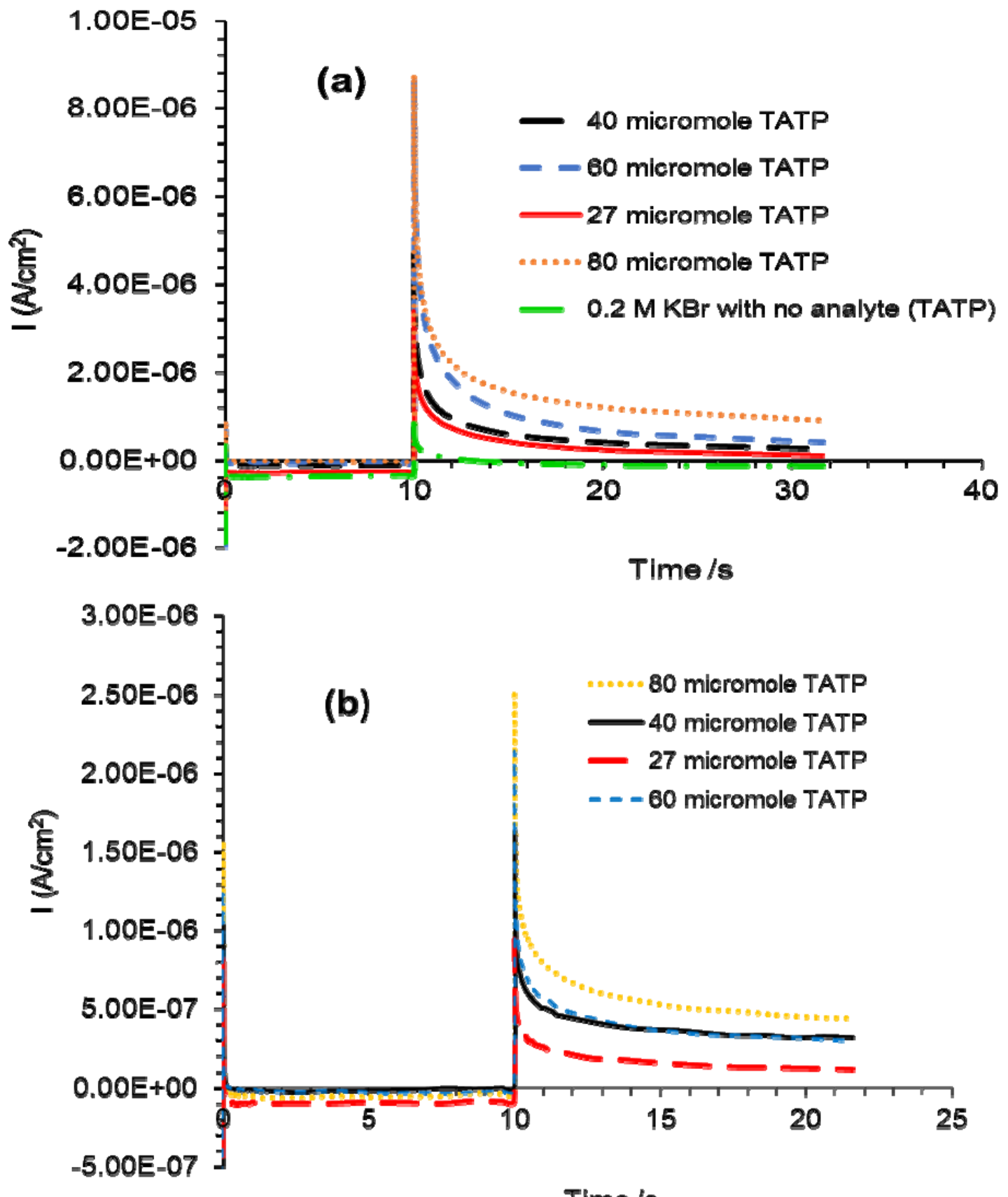

Figure 3. 


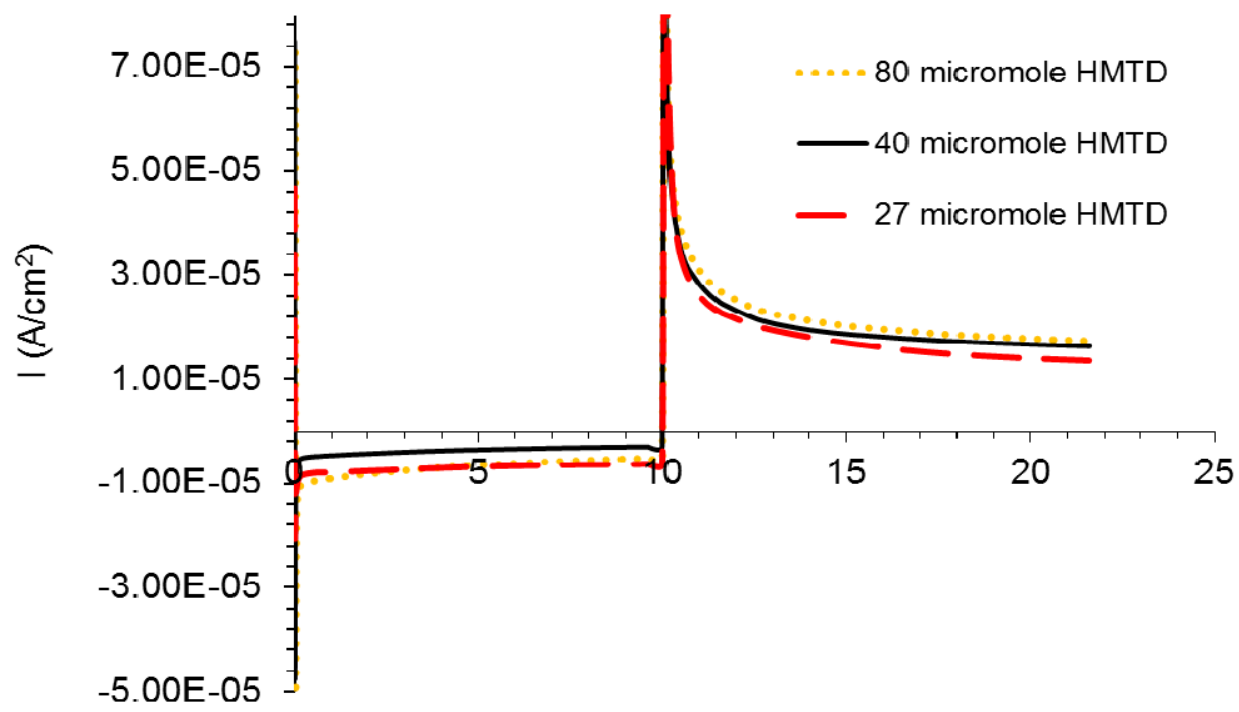

Time/s

Figure 4. 


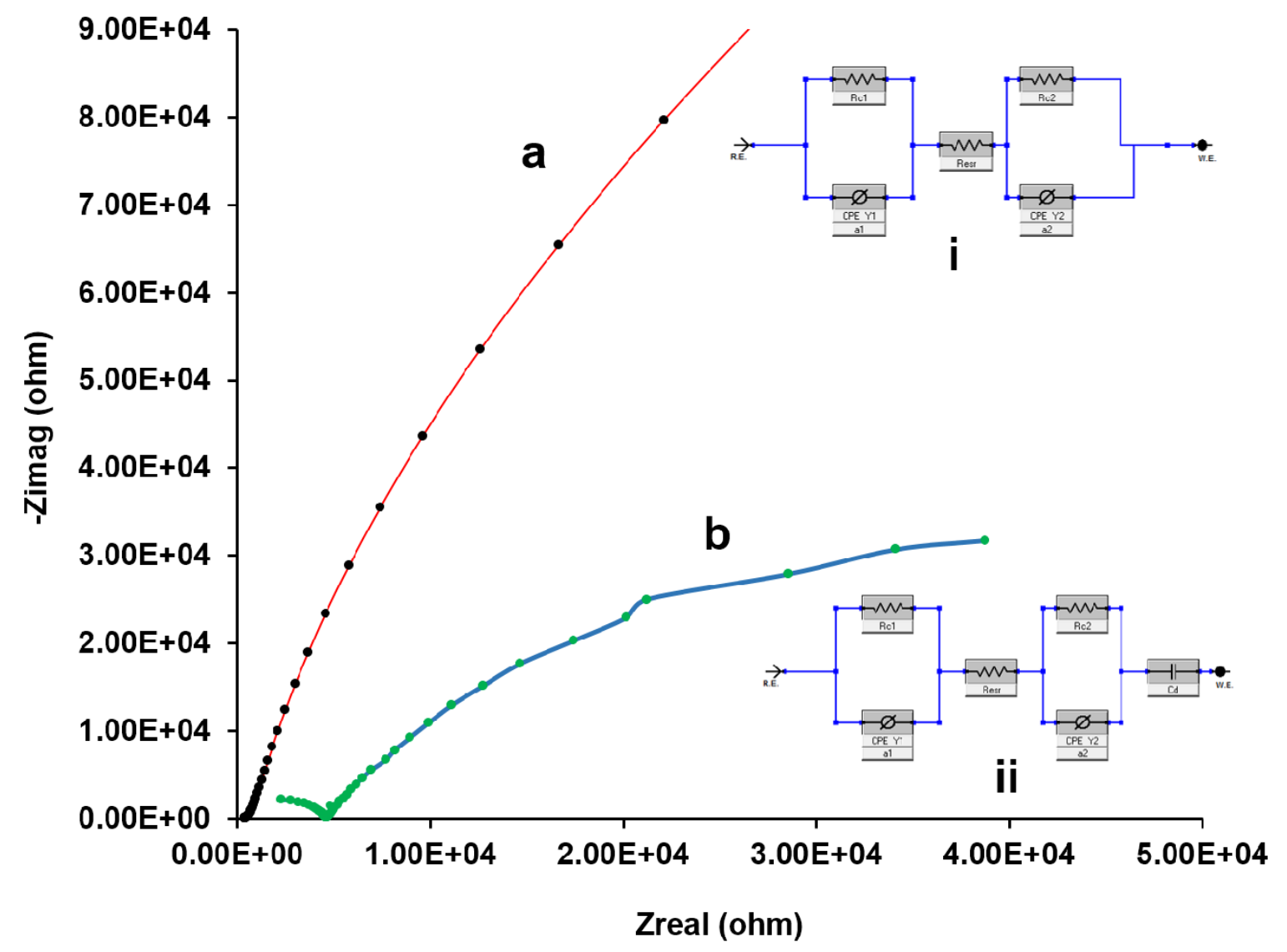

Figure 5. 